\title{
Advances in Ultra-weak Bioluminescence
}

\author{
Cheng Zhengyong ${ }^{1}$, Zhang Sheng ${ }^{1}$, Li Ying ${ }^{1,2}$, He Banggui ${ }^{1,2}$, Gu Wenjuan ${ }^{1,2, a}$ \\ ${ }^{1}$ Faculty of Mechanical and Electrical Engineering,Kunming University of Science and Technology, \\ Kunming, Yunnan 650500, China
}

${ }^{2}$ Engineering Research Center of paper packaging and Printing in Yunnan Province, Kunming, Yunnan 650500, China

aEmail: guwenjuan001@163.com

Keywords: Optimal Design ultra-weak bioluminescence; generation mechanism; application; detection

Abstract. Ultra-weak Bioluminescence is physiological phenomenon widely presenting in all biological life activities, which reflects the integrated vital signs of organisms. This paper analyses the current application of Ultra-weak Bioluminescence in biomedicine, agriculture, environmental science, food detection and other aspects based on discussing the generation mechanism and detection of ultra-weak Bioluminescence.

\section{Introduction}

As early as the 1920s, Ultra-weak Bioluminescence phenomenon was first discovered when Soviet scientists Gurwitsch research on onion root tip cell division mechanism. However, detection results of this radiation are not ideal and not repeatable due to the limitations of technical means at that time and therefore not recognized [1]. With photon counting technology advances, Italian Colli had further studies and indeed proved biological function of photon radiation at 1954[2]. Subsequently, research on UBL had developed comprehensively. To the 1960s, found that almost all life forms have ultra-weak luminescence.

\section{The mechanism of Ultra-weak Bioluminescence}

There are a lot of theoretical studies on the mechanism of Ultra-weak Bioluminescence and can be initially divided into two major aspects of physics and chemistry. It mainly has "metabolism luminescence" mechanism in chemistry and "coherent radiation" mechanism in physics [3].

"Metabolism luminescence" mechanism consider UBL is stochastic and spontaneous chemiluminescence in organism. This view is based on optical biochemistry and linked excited state to produce photon radiation with metabolism in life body. In this view, this kind of luminescence mainly stems from metabolic reactions, such as redox [4]. "Metabolism luminescence" mechanism include generation of reactive oxygen and control and can better explain the possibility of ultraweak luminescence caused by free radical. But it can not fully explain the ultra- weak luminescence of biological systems [3]. According to the "coherent radiation" mechanism, a part of photons of spontaneous and light-induced ultra-weak bioluminescence originate from a highly coherent electromagnetic field in biological systems, which is likely Communication foundation within living tissue[3].

\section{Detection of ultra-weak bioluminescence}

Intensity of Ultra-weak Bioluminescence is extremely weak, so we must use a very high detection sensitivity and low background noise photoelectric detection equipment to effectively detect.

At present, there are many kinds of detecting instrument used in detection of Ultra-weak bioluminescence. According to the structure and performance of the instrument can be divided into two categories: one is single photon counting detection system lead by the photomultiplier tube and 
provides time domain information of ultra-weak bioluminescence; Another is ultra-weak luminescence image detection system lead by micro-channel plate and a kind of micro-channel plate image intensifier as the core of ultra-high sensitivity imaging systems. The system can provide two-dimensional intensity distribution information and images of sample in real-time. The use of ultra-weak luminescence image detection device can visually monitor ultra-weak bioluminescence and changes in real-time on different parts of organisms, different metabolic stages or in different environments [5].

\section{Applications of ultra-weak Bioluminescence}

UBL has a broad and clear application prospect. UBL is a kind of natural biological function and inevitably associated with a variety of life processes. We can deeply understand of these life processes in greater detail through the measurement and analysis to UBL. Based on this various means (physical, chemical, medical, etc.) can also be used to artificially adjust UBL and control the life processes. UBL as an extremely sensitive indicator has been used in medicine, pharmacology, agriculture, environmental science and food science, etc. [6].

Ultra-weak bioluminescence had a great development in the recent twenty years, especially widely used in the biomedical field, has become an integral part of modern biotechnology. In particular application of luminescent immunoassay technology and luciferase reporter gene has been gradually formed an industry. Currently application of Ultra-weak bioluminescence technology in the biomedical field mainly includes cytology detection, molecular biology, hygiene and bio-detection sensors etc. Cytology detection mainly use luciferase bioluminescence caused by ATP in cell to count viable cell. At present, it has achieved rapidity, dynamic, single cell analysis. At the same time, some new cytology index related to bioluminescence and chemiluminescence also were found. Applications within the field of molecular biology major as a reporter gene and molecular hybridization, in recent years, some people launched real-time DNA sequencing technology; Detection in hygiene mainly uses molecular hybridization or bioluminescence caused by ATP to detect microbial contamination; in biosensors, the current major study is biological sensors based on immune response [7]. With luciferase gene as a report gene has been increasingly applied and studied, people began to explore and use the similar principle to build microbial sensors for environmental monitoring.

Currently, effects of moisture, light and temperature in grow on ultra-weak luminescence of the growth process is widely used in selection of resistant varieties (such as Drought-resistance, flood-resistance, cold-resistance, heat-resistance, salt-resistance etc.). For example, so far, there are more than ten kinds of drought indicators from morphology to physiology in the study of drought resistance, but these indicators are obviously deficient. Therefore, to explore whether the ultra-weak luminescence can be taken as physical indicator of identification of drought-resistance has important practical significance, and research in this area has made encouraging progress. Many studies show that drought-resistant varieties under simulated drought conditions have fast germination and intense ultra-weak luminescence. These studies involved mostly wheat, corn, soybeans and various vegetables.

Plant life needs during growth, such as moisture, temperature, light, nutrients, etc., is closely related with the growth activity and metabolism of plants. Metabolism activity is an important factor to ultra-weak bioluminescence, so plant life needs during growth can be manifested through its ultra-weak luminescence. When Plant life needs during growth is just met, metabolism of plant is fastest and its ultra- weak luminescence is also strongest. When life needs are not met or overly satisfied, metabolism of plant will reduce and its intensity of ultra-weak luminescence also decreases.

The field is currently at an initial stage. Studies have shown that some of the biology under participation of reactive oxygen species and pathological reactions can produce emitted photons, then ultra-weak luminescence show enhancement. Such ultra-weak bioluminescence is released when the biological body is pathological state and macrophages resist germs to produce toxicological response. At present, this is a new technology on research of plant disease resistance 
mechanisms.

Ultra-weak bioluminescence is very sensitive to many metal ions, so it can quantitatively determine concentration of $\mathrm{Ca}^{2}+, \mathrm{Cu}^{2}+, \mathrm{Zn}^{2}+, \mathrm{Mn}^{2}+, \mathrm{Fe}^{2}+$ and other elements. Ultra-weak bioluminescence is more sensitive than traditional chemical analysis for the detection of waste liquid, heavy metals and pathogens in industrial effluent [1].

Food security issue of agricultural products has become an important factor affecting social stability and sustainable agricultural development. Controlling and monitoring agricultural production and circulation process is foundation to ensure the safety of agricultural products. The development of simple, rapid, low-cost agricultural products safety testing technology is very important. Present study found that changes in emission intensity after spraying pesticide are faster than growth indicators. Such as $5 \times 10^{-8} \mathrm{~mol} / \mathrm{L}$ herbicides can cause significant changes in ultra-weak luminescence intensity, so it can be used to monitor pesticide pollution and pesticide poison to the environment or crops. By detecting ultra-weak luminescence of vegetable crops can directly reflect the impact of pollutants on physiological metabolism of vegetable crops, thus can provide a theoretical basis for detection of non-pollution vegetables and green vegetables. Living body can be detected in real time. We can quickly determine the type and status of environmental contaminants through change of the biological radiation spectrum caused by different types of pollution [8].

Experimental results show that the level (only $0.2 \mu \mathrm{g} / \mathrm{m}^{3}$ ) of $\mathrm{SO}_{2}$ in the atmosphere can affect the ultra-weak bioluminescence of some plants, so we can analyze quantitatively the content of $\mathrm{SO}_{2}$ in the atmosphere through detecting ultra-weak bioluminescence. Before the earthquake, some increased substance content in the atmosphere can make ultra-weak bioluminescence of some plants change obviously. Detecting this change may provide a basis for earthquake prediction [9].

\section{Conclusion}

Ultra-weak bioluminescence as an extremely sensitive indicator in life processes has been used in many fields and will be more widely used. It is an encouraging development. But it should be noted on the fundamental research for UBL there are many important unresolved issues, such as biological mechanisms of ultra-weak bioluminescence production. In addition, from the perspective of quantum science, UBL photon statistics should contain a wealth of biological information. How to get the biological information bringing practical value by statistics and measurements of UBL photon are important theoretical and practical problem to be solved. A large number of research data show that the scope of research in plants for UBL is quite narrow, and mostly concentrated on field crops. Studies in the trees and fruit trees are relatively small and mostly concentrate in drought-resistance research. Therefore, we also need to make a lot of research work to mechanism, application etc. of UBL, in order to better service the development of agriculture and other sciences. Although there are many deficiencies in the current study for UBL, but UBL show a bright future due to revealed life sciences proposition.

\section{Acknowledgement}

In this paper, the research was sponsored by the Personnel Training Foundation of Yunnan Province (Project No. KKSY201201051).

\section{References}

[1] Yuan Zuoqing. Research progress on ultra-weak bioluminescence [J].Journal of Anhui Agricultural Sciences, 2008,36(8):3092 3094

[2] Colli L, Facchini U, Zhou Yu et al. Further measurements on the bioluminescence of the seedlings[J]. Experientia, 1955,11(1):479 481 
[3] Wang Weijiang, Han Junying. Advance in the study of ultra-weak bioluminescence mechanisms and detecting methods[J]. Journal of Guangdong University of Technology, 2000,17(1):49 54

[4] Li Shuli, Zheng Xinliang, Ren Zhaoyu. The progress of ultraweak photon emission from living sysrems[J]. Laser Journal, 2004,25(4):4 6

[5] Wang Weijiang, Xing Da, Tan Shici. Detection of plant ultraweak bioluminescence[J]. Journal of Optoelectronics•Laser, 2000,11(4):427 430

[6] Zhang Xinhua, Yang Hongqing. Ultraweak bioluminescence[J]. Journal of Shandong agricultural university, 2003,34(4):605 608

[7] Yang Xiaolin. The development in applications of bioluminescence \& chemiluminescence to bio-medical science[J]. Acta biophysica sinica, 2000,16(3):10 18

[8] Zhang Juping, Zhang Xingzhi,Gong Zhenhui. Application of ultraweak bioluminescence in vegetable research[J]. Chinese agricultural science bulletin Acta Optica Sinica, 2003,23(12):1424 1428

[9] Xue Hongfu. On recent advances in the field of ultraweak photon emission from biological system[J].Physical experiment of college, 1996,9(4):9〜11 